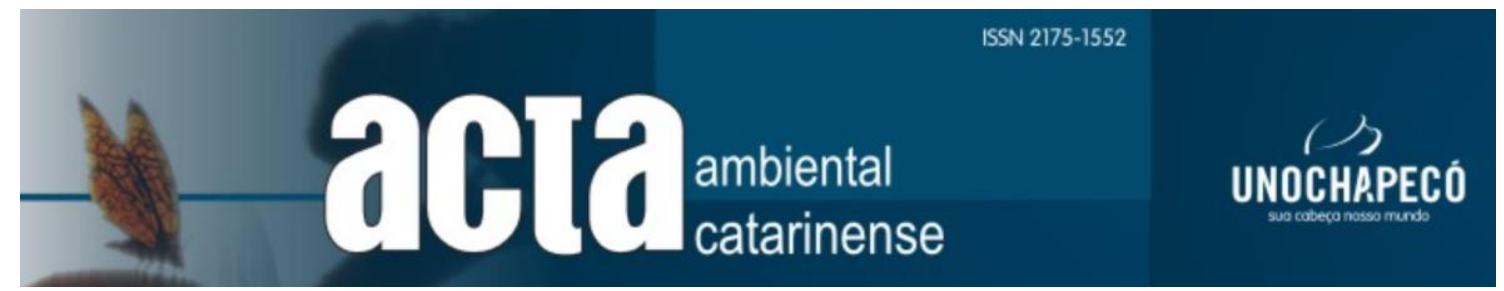

\title{
EFEITO SINERGÉTICO DO BTI E PREDAÇÃO SOBRE A MORTALIDADE DE LARVAS DO MOSQUITO Aedes aegypti (LINNAEUS, 1762)
}

\author{
Gabriel de Carvalho1; Gilberto Dinis Cozzer2*; Renan de Souza Rezende³ Jacir Dal Magro4; Daniel Albeny \\ Simões $^{5}$
}

\begin{abstract}
${ }^{1}$ Discente do curso de Graduação em Ciências Biológicas pela Universidade Comunitária da Região de Chapecó.
${ }^{2}$ Mestrando do Programa de Pós-Graduação em Ciências Ambientais pela Universidade Comunitária da Região de Chapecó.

${ }^{3}$ Doutor em Ecologia pela Universidade Federal de Santa Catarina. Professor permanente do Programa de Pós-Graduação em Ciências Ambientais da Universidade Comunitária da Região de Chapecó.

${ }^{4}$ Doutor em Química pela Universidade Federal de Santa Catarina. Professor permanente do Programa de Pós-Graduação em Ciências Ambientais da Universidade Comunitária da Região de Chapecó.
\end{abstract}

${ }^{5}$ Doutor em Entomologia pela Universidade Federal de Viçosa.

*E-mail: pinocozzer.ps@unochapeco.edu.br

\section{Resumo}

O objetivo deste trabalho foi avaliar o efeito do uso de larvas predadoras da ordem Odonata (família Libellulidae) associado ao BTI na redução larval de mosquitos. Nossa hipótese testada busca responder se haveria aumento na mortalidade de larvas de A. aegypti causada pelo efeito sinergético do BTI juntamente com o predador. Os microcosmos constituíam-se de recipientes com capacidade para $300 \mathrm{~mL}$ contendo $100 \mathrm{~mL}$ de água com densidades de 100, 200, e 300 larvas de A. aegypti e uma larva do predador. Os tratamentos, BTI e BTI + Predador receberam 0,01ppm de BTI. O controle recebeu apenas as larvas de A. aegypti. Os resultados mostraram um efeito significativo do tratamento BTI + Predador na densidade de $1 \mathrm{larva} / \mathrm{mL}$, enquanto nas densidades de 2 e 3 larvas/mL o mesmo efeito não foi observado, corroborando parcialmente a hipótese de efeito sinergético do BTI e predação na mortalidade larval. O aumento da densidade larval aumentou a taxa de predação, enquanto o efeito do BTI sobre a mortalidade diminuiu. Nossos resultados mostram que o efeito sinergético existe, mas que fatores dependentes da densidade podem ocultar tal efeito.

Palavras-chave: Odonata, Bacillus thuringiensis israelensis, predação.

\begin{abstract}
This study objective was to evaluate the effect of use of predatory Odonata (family Libellulidae) larvae associated with BTI on larval reduction of mosquitoes. Our tested hypothesis seeks to answer whether there would be an increase in the mortality of A. aegypti larvae caused by the synergistic effect of BTI along with the predator. The microcosms consisted of $300 \mathrm{~mL}$ containers containing $100 \mathrm{~mL}$ of water with densities of 100, 200, and 300 larvae of A. aegypti and one predator larva. The BTI and BTI + Predator treatments received $0.01 \mathrm{ppm}$ BTI. Control received only A. aegypti larvae. The results showed a significant effect on BTI + Predator treatment in the density of 1 larva / mL, while in the densities of 2 and 3 larvae / $\mathrm{mL}$ the same effect was not observed, partially corroborating the hypothesis of BTI and predation synergistic effect in larval mortality. Increasing larval density increased predation rate, while the effect of BTI on mortality decreased. Our results show that the synergistic effect exists, but that density-dependent factors may hide such effect.
\end{abstract}

Keywords: Odonata, Bacillus thuringiensis israelensis, predation. 


\section{Introdução}

A correta destinação final de recipientes artificiais com capacidade para conter água (ex. copos plásticos, pneus, garrafas, latas) é um problema em grande parte das cidades brasileiras (FORATTINI e BRITO, 2003). O descarte destes materiais em locais inapropriados e com exposição às condições climáticas (ex. chuva), contribui para a criação de micro-hábitats aquáticos (FORATTINI e BRITO, 2003). Estes micro-habitats aquáticos podem servir como local de desenvolvimento para várias espécies de macroinvertebrados, como o mosquito Aedes aegypti (Linnaeus, 1762) (MERRITT et al., 1992; LOPES et al., 1993). Transmissor de diversas arboviroses (ex. dengue, Chikungunya e Zika) (JOUBERT e O’NEILL, 2017; PLIEGO-PLIEGO et al., 2020), as fêmeas desta espécie utilizam com sucesso estes locais para depositar seus ovos e para que as formas larvais possam se desenvolver (LOPES et al., 1993; CONSOLI e LOURENÇO-DEOLIVEIRA, 1994; CARLSON et al., 2020).

Quando mais de uma espécie coloniza um micro-habitat aquático é esperado que interações tróficas aconteçam (ALBENY-SIMÕES et al., 2015). Redes de interações tróficas geralmente exibem a configuração composta por organismos produtores, consumidores, predadores $\mathrm{e}$ microrganismos detritívoros (WALKER et al., 1991; KITCHING, 2000; ALBENY-SIMÕES et al., 2015). Produtores primários fototróficos convertem energia luminosa em energia química, incorporando e incrementando a quantidade de matéria presente no sistema (FIGUEIREDO, 2017). Detritívoros quebram moléculas maiores e complexas, disponibilizando nutrientes para os consumidores (MERRIT et al., 1992). Consumidores são formados por um extenso grupo de macroinvertebrados, os quais se podem se alimentar tanto da matéria orgânica que adentra o sistema como dos microrganismos decompositores dessa matéria orgânica (MERRIT et al., 1992). Os predadores são usualmente generalistas e desempenham importante papel na estruturação das comunidades (ZARET, 1980).

Um dos predadores comumente encontrados em microhábitats aquáticos são as larvas de Odonata, onde capturam e consomem uma variada gama de organismos aquáticos, inclusive larvas de mosquitos (Diptera: Culicidae) (MIURA e TAKAHASHI, 1988; SEBASTIAN et al., 1990; ANDRADE e SANTOS, 2004; QUIROZ-MARTÍNEZ et al., 2005; FULAN et al., 2014). Por causarem a redução populacional de suas presas através do consumo direto, são reconhecidas como armadilhas ecológicas, contribuindo para o controle de algumas espécies de mosquitos veiculadores de doenças (KRISTAN III, 2003). Dada à importância desses microhabitats para fomentar o desenvolvimento de mosquitos em ambiente urbano, diversas estratégias de controle têm sido utilizadas como ferramentas para controlar formas imaturas e adultos (TAUIL, 2002).

O controle populacional de mosquitos utilizando-se predadores é uma alternativa ao controle químico, especialmente quando o uso de produtos de origem química seleciona genes que conferem resistência ao organismo alvo (TAUIL, 2002; LIMA et al., 2006; BRAGA e VALLE, 2007), além de todos os prejuízos causados ao meio ambiente (BASKAR et al., 2018) e a saúde humana (GOVINDARAJAN et al., 2018). Da mesma forma, o uso dos esporos letais da bactéria Bacillus thuringiensis israelensis (BTI) é outro método alternativo amplamente utilizado no Brasil para o controle de larvas de A. aegypti, cujas intervenções apresentam alta efetividade (POLANCZYK et al., 2003; BARRETO et al., 2008; ALTO e LORD, 2016). O BTI é um inseticida biológico seletivo para a ordem díptera (Sumitomo Corporation ${ }^{\circledR}$ ). As toxinas liberadas pela digestão dos esporos ingeridos pelas larvas causam a morte do indivíduo por lesões na parede do epitélio intestinal (GILL et al., 1992). Mesmo em concentrações muito inferiores à recomendada pelo fabricante, o BTI tem se mostrado bastante eficiente (ALTO e LORD, 2016).

Larvas de mosquitos constituem uma importante parte da dieta para predadores aquáticos (HERSHEY et al., 1998). Partindo desta premissa, este trabalho tem por objetivo verificar se existe algum efeito aditivo (BTI + predação) na redução populacional de mosquitos em um sistema onde a predação está ocorrendo. Como organismos modelo utilizamos larvas do mosquito Aedes aegypti e larvas de libélulas predadoras. O presente estudo testa a hipótese de que se o BTI é seletivo para a ordem díptera, a adição ao sistema de uma libélula predadora junto com o BTI irá promover uma maior mortalidade de larvas de mosquitos do que simplesmente o BTI e o predador sozinhos.

\section{Material e Métodos}

\subsection{Obtenção dos Insetos}

\subsubsection{Predadores}

As larvas de libélulas foram coletadas na Gleba 2 $\left(27^{\circ} 6^{\prime} 14.31 " \mathrm{~S} / 52^{\circ} 45^{\prime} 33.51^{\prime \prime} \mathrm{W}\right)$ da Floresta Nacional de Chapecó (FLONA), situada no município de Guatambu, Santa Catarina. Para a coleta das larvas de libélula, foram utilizadas quatro caixas d'água com capacidade para 200L e dezesseis baldes com capacidade para 15L, que foram espalhados sistematicamente pela unidade de conservação. As larvas de libélula foram capturadas através da submersão de uma rede de plâncton $(120 \mu \mathrm{m})$ nestes depósitos artificiais. As larvas foram

individualmente acondicionadas em frascos tipo Nalgon com capacidade para $300 \mathrm{~mL}$ e levadas para o laboratório. Em seguida, foram transferidas para copos plásticos (capacidade $80 \mathrm{~mL}$ ) contendo $50 \mathrm{~mL}$ de água de água oriunda da cisterna que abastece o Laboratório de Entomologia Ecológica (portanto livre de cloro) e deixadas em aclimatação por um período de 72 horas. Para evitar o desenvolvimento rápido as larvas de libélula foram diariamente alimentadas com cinco larvas de $A$. aegypti de $3^{\circ}$ e $4^{\circ}$ instares até que o experimento de microcosmos fosse iniciado.

\subsubsection{Presas}

As larvas de Aedes aegypti utilizadas no experimento foram oriundas da colônia de mosquitos existente no Laboratório de Entomologia Ecológica (LABENT-Eco) da Universidade 
Comunitária da Região de Chapecó. A eclosão dos ovos foi feita através da imersão dos papéis de oviposição em bandejas $(30 \times 15 \times 5 \mathrm{~cm})$ contendo 1 litro de água oriunda de uma cisterna que abastece o LABENT-Eco, portanto sem tratamento químico. A densidade larval variou de 500 à 1000 larvas por bandeja. As larvas foram alimentadas com ração para peixes Tetra Fin ${ }^{\circledR} 0,1 \mathrm{~g} / \mathrm{L}$ e mantidas nas bandejas até atingirem o $3^{\circ}$ instar. A sala de criação foi mantida a $27^{\circ} \mathrm{C} \pm 3$, umidade relativa $80 \%$ e fotoperíodo de $12: 12 \mathrm{~h}$ de claro:escuro.

\subsection{Desenho Experimental}

Os microcosmos experimentais foram constituídos de copos plásticos com capacidade para $300 \mathrm{~mL}$, contendo $100 \mathrm{~mL}$ de água e diferentes densidades de larvas de A. aegypti $\left(100,200\right.$ e 300 larvas) entre $3^{\circ}$ e $4^{\circ}$ ínstares. Ao todo foram testados 4 tratamentos, com seis réplicas por tratamento. Dois tratamentos contavam com a presença do predador, denominados Tratamento Predador e Tratamento Predador + BTI, e dois tratamentos não receberam predador, denominados Tratamento BTI e Controle. Os tratamentos com o predador presente, receberam uma larva de libélula, independentemente da densidade larval de presas. Os tratamentos com BTI receberam Bti - Vectobac ${ }^{\circledR}$ na concentração de 0,01 parte por milhão (ppm) e o controle era constituído apenas por água filtrada. A concentração de BTI utilizada de 0,01 ppm seguiu o trabalho de Charbonneau et al. 1994. Ao final de 24 horas foram contadas as larvas vivas remanescentes e subtraído do número inicial, contabilizando assim o número de larvas de A. aegypti mortas ou que foram predadas. Para o tratamento Predador + BTI não foram contabilizadas em separado as mortes causadas por predação ou por BTI, apenas consideramos a mortalidade final por predação e BTI ao final das $24 \mathrm{~h}$ experimentais.

\subsubsection{Diluição do BTI}

Para obter a concentração de $0,01 \mathrm{ppm}$, foi depositado $1000 \mathrm{~mL}$ de água filtrada a um copo Becker de vidro, pesado $0,01 \mathrm{~g}$ de BTI utilizando balança de precisão $( \pm 0,0001 \mathrm{~g})$ e adicionado a este mesmo copo Becker. Após diluir por 4 min foi retirado $1 \mathrm{ml}$ desta concentração estoque e diluído novamente durante 4 minutos em outro copo Becker contendo $1000 \mathrm{~mL}$ de água filtrada. Após este procedimento foi retirado $100 \mathrm{~mL}$ desta concentração $(0,01 \mathrm{ppm})$ adicionado nas réplicas dos tratamentos com presença do BTI.

\subsection{Análises Estatísticas}

Modelo linear generalizado (GLM) fatorial foi utilizado para análise dos efeitos de diferentes tratamentos experimentais (BTI, Controle, Predador + BTI e Predador), densidade de larvas e interação entre ambos (variáveis explicativas) sobre a porcentagem de larvas de A. aegypti mortas ao final de $24 \mathrm{~h}$ de exposição (variáveis de resposta; Crawley 2007). O GLM foi realizado com base na distribuição Binomial (link $=$ logit, teste $=$ Chisq) e ajustado para QuaseBinomial (link $=$ logit, teste $=$ Chisq) para corrigir sobre- dispersão. Foi realizado o teste post hoc de Análise de Contraste para verificar as diferenças entre os tratamentos e densidade de larvas. As análises e gráficos foram realizadas no programa estatístico livre R (R Development Core Team, 2017).

\section{Resultados}

\subsection{Efeitos dos Tratamentos Sobre a Mortalidade}

Houve efeito significativo dos tratamentos sobre a mortalidade larval nas três densidades testadas (Tabela 1) larvas. $\mathrm{Na}$ densidade 100 larvas houve maior mortalidade no tratamento Predador + BTI (93.5 \pm 3.52$)$, seguido dos tratamentos BTI $(70.67 \pm 3.2)$ e Predador $(70 \pm 6.73)$. O controle não apresentou mortalidade larval (Figura 1b). As densidades de 200 e 300 larvas não apresentaram diferença significativa entre os tratamentos Predador $(147.17 \pm 7.41$; $126.17 \pm 20.26)$ e Predador + BTI $(131.67 \pm 9.88 ; 137.83 \pm$ 20.55), e ambos apresentaram maior mortalidade que tratamento BTI $(79.17 \pm 4.9 ; 30 \pm 4.39)$ (Figuras $1 \mathrm{~b}$ e $1 \mathrm{c})$.

Tabela 1. Influência do tratamento, densidade larval e interação entre fatores (Tratamento $x$ Densidade) na porcentagem de mortalidade larval de A. aegypti ao final de $24 \mathrm{~h}$ de exposição. Graus de liberdade (GL), desvio residual total (Desvio) e em porcentagem (\% Desvio) e significância (Pr) do modelo GLM testado. Teste post hoc de Análise de Contraste para verificar as diferenças entre os tratamentos e densidade de larvas.

\begin{tabular}{lccccc}
\hline & GL & Desvio $\%$ Desvio & Pr $(>$ Chi $)$ & Análise de contraste \\
\hline \hline Nulo & 107 & 84 & 100.0 & & \\
Tratamento & 3 & 61.6 & 73.3 & $<0.001$ & Controle $=$ BTI $<$ PBTI $=$ PRED \\
Densidade & 1 & 6.6 & 7.9 & $<0.001$ & D $300<$ D $200<$ D 100 \\
Tratamento x Densidade & 3 & 3.3 & 3.9 & $<0.001$ & \\
Resíduo & 100 & 12.5 & 14.9 & & \\
\hline
\end{tabular}

\subsection{Efeitos das Densidades Sobre a Mortalidade}

Houve efeito significativo das densidades larvais (larvas $/ \mathrm{mL}$ ) sobre a mortalidade de larvas entre os tratamentos Predador, Predador + BTI, BTI e controle (Tabela 1). No tratamento Predador houve aumento da predação com o aumento da densidade larval (Figura 1c). Entretanto, o número médio de presas consumidas (aproximadamente 135 larvas) estabilizou com o aumento da densidade larval, independente desta ser 2 ou 3 larvas/mL (Figura 1c). No tratamento BTI, o número de larvas mortas estabeleceu uma relação negativa com a densidade de larvas por $\mathrm{mL}$. Houve diminuição significativa no número de larvas mortas pelo BTI com o acréscimo da densidade. Para o tratamento Predador + BTI, ocorreu efeito positivo da densidade larval sobre a mortalidade de larvas de A. aegypti (Figura 1c). Houve aumento no número de larvas mortas pelo tratamento Predador + BTI da densidade de 1 para 3 larvas/mL. 

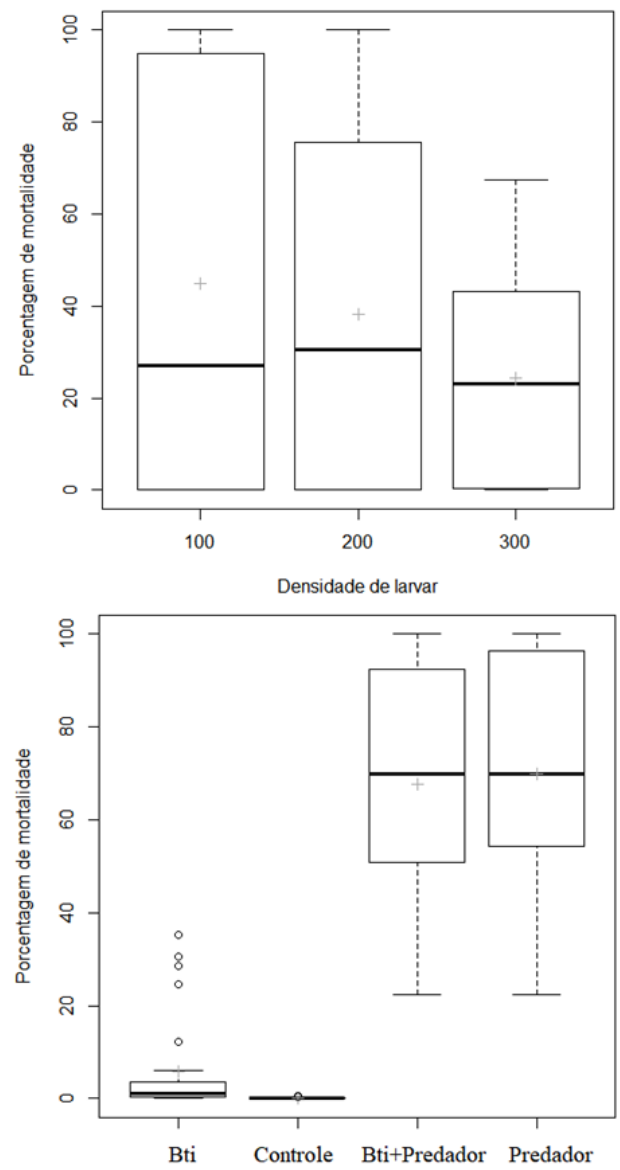

(b)

(c)

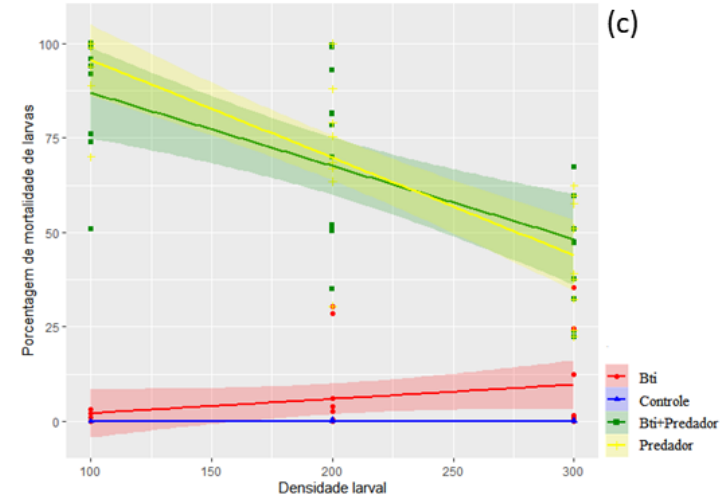

Figura 1. Porcentagem de mortalidade de larval de A. aegypti mortas ao final de $24 \mathrm{~h}$ de exposição entre as diferentes densidade larvais (a), tratamentos (b) e interação entre densidade e tratamentos (c). Nas figuras "a" e "b" as caixas representam os quartis, a linha em negrito representa a mediana, a cruz cinza é a média, a linha tracejada vertical representa os limites superior e inferior e circulo os valores extremos (outliers). Na figura "c" as linhas representam a variação entre os tratamentos e as áreas coloridas das respectivas cores os intervalos de confiança de $95 \%$ dos modelos.

\section{Discussão}

O experimento mostra efeito significativo dos tratamentos afetando a mortalidade das larvas de A. aegypti nas três densidades testadas (100, 200 e 300 larvas) (Tabela 1). Na densidade de 100 larvas o tratamento Predador + BTI foi o que apresentou maior mortalidade de larvas em comparação aos demais tratamentos (Predator, BTI e Controle). Resultado que corrobora com a hipótese de efeito sinergético entre BTI e predador na redução do número de larvas de A. aegypti. O aumento na densidade larval para 200 e 300 larvas dificulta a observação da existência de efeito sinergético, uma vez que há a redução da pressão de predação exercida pelo predador sobre as larvas de $A$. aegypti com o aumento da densidade. Ao mesmo tempo, há a diluição da concentração de BTI por um número $2 \mathrm{x} / 3 \mathrm{x}$ maior de larvas, reduzindo a quantidade de esporos ingeridos e, consequentemente, a mortalidade larval (Figura 1b e 1c).

Houve aumento do número de larvas predadas com o aumento da densidade larval, com um consumo de aproximadamente 135 larvas de A. aegypti em 24 horas, nas densidades maiores (Tabela 2). Larvas de libélula são predadores do tipo senta e espera (SOARES et al., 2008), logo, ambientes mais adensados promovem uma maior taxa de encontro entre predador e presa, o que resulta em um maior número de eventos de predação (SUNAHARA et al., 2002). Albeny-Simões et al. (2012) mostraram que o aumento da densidade larval, promovido pela alteração nas dimensões do hábitat, resulta em um aumento da taxa de captura de larvas de A. aegypti pelo predador Toxorhynchites theobaldi (Diptera: Culicidae). Weterings et al. (2015), em um ensaio realizado com larvas de libélulas e A. aegypti observaram aumento substancial na predação diária quando a densidade larval é aumentada de 10 para 50 larvas oferecidas.

O aumento na densidade larval no sistema promove, além do aumento da captura de presas pelo predador, a diminuição na letalidade do BTI (Figura 1a e 1b). O aumento da densidade larval no sistema diminui a quantidade de BTI por indivíduo o que resulta em menor letalidade por diminuição da concentração individual do bioinseticida (MULLA et al., 1990; BECKER et al., 1992). O mecanismo de ação do BTI sobre o A. aegypti, após a ingestão dos esporos da bactéria adicionados à água, ocorre pela liberação das proteínas tóxicas presentes na estrutura química dos esporos durante o processo de digestão pela larva (GILL et al., 1992). Essas toxinas danificam as paredes do epitélio intestinal das larvas, interrompendo o peristaltismo e causando a morte (GILL et al., 1992).

Embora não tenhamos encontrado efeito sinergético no tratamento Predador + BTI nas densidades de 2 e 3 larvas $/ \mathrm{mL}$, o efeito aditivo do BTI associado ao Predador no sistema ocasionou mais mortalidade do que o BTI isoladamente (Figura 1b; Tabela 2). A mortalidade larval causada pelo BTI em diferentes densidades, demonstra que a quantidade de BTI ingerida por cada indivíduo é menor quanto mais adensado é 
um sistema (Tabela 2). Com uma menor concentração de BTI por indivíduo, o contato de cada larva com a quantidade de BTI necessária para ter efeito letal fica prejudicado. Desse modo, 24 horas de exposição não foi tempo suficiente para provocar mortalidade ao ponto de ter efeito adicional de mortalidade do tratamento Predador + BTI sobre o tratamento Predador nas densidades de 1 e 3 larvas $/ \mathrm{mL}$.

Esses resultados são inovadores pois trazem abordagens ecológicas do entendimento dos efeitos dependentes da densidade sobre a ação direta (sobre larvas) e ação indireta (sobre predadores) do BTI em sistemas artificiais. O BTI não possui ação tóxica ou letal sobre os níveis tróficos superiores (predadores) testados neste experimento. Contudo para que possamos entender os efeitos sinergéticos da predação associado a presença de outros métodos que tenham efeitos subletais sobre as populações de mosquitos, precisamos elucidar em estudos futuros os efeitos da densidade e também do tempo exposição dos organismos ao composto testado.

\section{Agradecimentos}

Os autores agradecem à Universidade Comunitária da região de Chapecó pela disponibilização dos laboratórios e equipamentos necessários à execução deste trabalho e ao Projeto CNPq 421288/2017-5 pelo apoio financeiro.

\section{Referências}

ALBENY-SIMÕES D, CASSOL AS, BREAUX JA, ANDRADE MR, LIMA E, VILELA E. Efficiency of the induced mating technique for Toxorhynchites theobaldi (Diptera, Culicidae). Revista Brasileira de Entomologia, v. 59, p. 65-67, 2015.

ALBENY-SIMÕES D, ROSA CS, KRÜGER RF, ATAÍDE LMS, VILELA EF. Variação da predação de larvas de Aedes aegypti por larvas de Toxorhynchites violaceus (Diptera: Culicidae) de acordo com dimensões do hábitat. BioAssay, v. 7, n. 4, 2012.

ALTO BW, LORD CC. Transstadial effects of Bti on traits of Aedes aegypti and infection with Dengue Virus. Neglected Tropical Diseases, v. 10, 2016.

ANDRADE CFS, SANTOS LU. O uso de predadores no controle biológico de mosquitos, com destaque aos Aedes. Campinas: PPGP, 2004. Disponível em: <http://www2.ib.unicamp.br/profs/eco_aplicada/arquivos/arti gos_tecnicos/C\%20B\%20de $\% 20$ mosquitos $\% 20 \mathrm{eu}+\mathrm{lu} \% 2020$ 04.pdf>. Acesso em: 20 de maio de 2018.

BARRETO CE, GUEDES RN, DE SOUZA RCP. Avaliação da eficácia do Bacillus thuringiensis var. israelensis no controle de formas imaturas do Aedes (Stegomyia) aegypti (Linnaeus, 1762) em ambiente de laboratório. EntomoBrasilis, v. 1, p. 10-13, 2008.

BASKAR K, SUDHA V, NATTUDURAI G, IGNACIMUTHU S, DURAIPANDIYAN V, JAYAKUMAR M, AL-DHABI NA, BENELLI G. Larvicidal and repellent activity of the essential oil from Atalantia monophyllaon three mosquito vectors of public health importance, with limited impact on non-target zebra fish. Physiological and Molecular Plant Pathology, v. 101, p. 197-201, 2018.

BECKER N, ZGOMBA M, LUDWIG M, PETRIC D, RETTICH F. Factors influencing the activity of Bacillus thuringiensis var. israelensis treatments. Journal of the American Mosquito Control Association, v. 8, p. 285-289, 1992.

BRAGA IA, VALLE D. Aedes aegypti: vigilância, monitoramento da resistência e alternativas de controle no Brasil. Epidemiologia e Serviços de Saúde, v. 16, p. 295302, 2007.

CARLSON JS, SHORT SM, ANGLERÓ-RODRÍGUEZ YI, DIMOPOULOS G. Larval exposure to bacteria modulates arbovirus infection and immune gene expression in adult Aedes aegypti. Developmental and Comparative Immunology, v. 104, p. 1 - 8, 2020.

CHARBONNEAU CS, DROBNEY RD, RABENI CF. Effects of Baccilus thuringiensis var. israelensis on nontarget benthic organisms in a lentic habitat and factors affecting the efficacy of the larvicide. Environmental Toxicology and Chemistry, v. 13, n.2, p. 267-279, 1994.

CONSOLI RAGB, LOURENÇO-DE-OLIVEIRA R. Principais mosquitos de importância sanitária no Brasil. Rio de Janeiro: FIOCRUZ, 1994. 215 p.

CRAWLEY MJ. The R Book. Chichester: John Wiley e Sons Inc.; 2007.

FIGUEIREDO GM. O plâncton na elaboração de modelos Ecopath: importância, particularidades na coleta, análise e parametrização. In: ANGELINI R, ARAÚJO JN, FALCÃO APC. (Ed.) Modelagem Ecossistêmica para integração e manejo na Bacia de Campos (Atlântico Sudoeste), Atlântico Sudoeste. Rio de Janeiro: Elsevier. Habitats, v. 8. p. 37-62, 2017.

FORATTINI OP, BRITO M. Reservatórios domiciliares de água e controle do Aedes aegypti. Revista Saúde Pública, v. 37, p. 676-687, 2003. 
FULAN JÂ, SANTOS LR, FERREIRA L. Observações sobre a criação de ninfas de Brechmorhoga Kirby, 1894 (Insecta: Odonata) em condições artificiais. Revista Brasileira de Biociências, v. 12, p. 206-209, 2014.

GILL SS, COWLES EA, PIETRANTONIO PV. The mode of action of Bacillus thuringiensis endotoxins. Annual review of entomology, v. 37, p. 615-634, 1992.

GOVINDARAJAN M, RAJESWARY M, SENTHILMURUGAN S, VIJAYAN P, ALHARBI NS, KADAIKUNNAN S, KHALED JM, BENELLI G. Larvicidal activity of the essential oil from Amomum subulatum Roxb. (Zingiberaceae) against Anopheles subpictus, Aedes albopictus and Culex tritaeniorhynchus (Diptera: Culicidae), and non-target impact on four mosquito natural enemies. Physiological and Molecular Plant Pathology, v. 101, p. 219-224, 2018.

HERSHEY AE, LIMA AR, NIEMI GJ, REGAL RR. Effects of Bacillus thuringiensis israelensis (Bti) and methoprene on nontarget macroinvertebrates in Minnesota wetlands. Ecological Applications, v. 8, p. 41-60, 1998.

JOUBERT DA, O'NEILL SI. Comparison of stable and transient Wolbachia infection models in Aedes aegypti to block dengue and West Nile viruses. PLoS Negl. Trop. Dis, v. 11, 2017.

KITCHING RL. Food webs and container habitats: the natural history and ecology of phytotelmata. Cambridge University Press, Cambridge, UK, 2000.

KRISTAN III, WB. The role of habitat selection behavior in population dynamics: source-sink systems and ecological traps. Oikos, v. 103, p. 457-468, 2003.

LIMA EP, OLIVEIRA FILHO AMD, LIMA JWDO, RAMOS JÚNIOR AN, CAVALCANTI LPDG. Resistência do Aedes aegypti ao temefós em municípios do estado do Ceará. Revista da Sociedade Brasileira de Medicina Tropical, v. 39, p. 259-263, 2006.

LOPES J, SILVA MA, BORSATO AM, OLIVEIRA VD, FRANSISCO JDA. Aedes (Stegomyia) aegypti $L$. e a culicideofauna associada em área urbana da região sul, Brasil. Revista de Saúde Pública, v. 27, n. 5, p. 326-333, 1993.

MERRITT RW, DADD RH, WALKER ED. Feeding behavior, natural food, and nutritional relationships of larval mosquitoes. Annual review of entomology, v. 37, p. 349374, 1992.

MIURA T, TAKAHASHI P. A laboratory study of predation by danselfly nymphs, Enallagma civile, upon mosquito larvae, Culex tarsalis. J. American Mosquito Control Association, v. 4, p. 129-131, 1988.

MULLA MS, DARWAZEH HA, ZGOMBA M. Effect of some environmental factors on the efficacy of Bacillus sphaericus (2362) and Bacillus thuringiensis (H-14) against mosquitoes. Bulletin of the Society for Vector Ecology, v. 15, p. 166-175, 1990.

PLIEGO-PLIEGO E, VASILIEVA O, VELÁZQUEZCASTRO J, COLLAR AF. Control strategies for a population dynamics model of Aedes aegypti with seasonal variability and their effects on dengue incidence. Applied Mathematical Modelling, v. 81, p. 296-319, 2020.

POLANCZYK RA, GARCIA MO, ALVES SB. Potential of Bacillus thuringiensis israelensis for controlling Aedes aegypti. Revista de Saúde Pública, v. 37, n. 6, p. 813-816, 2003.

QUIROZ-MARTÍNEZ H， RODRÍGUEZ-CASTRO VA, SOLÍS-ROJAS C, MALDONADO-BLANCO MA. Predatory capacity and prey selectivity of nymphs of the dragonfly Pantala hymenea. Journal of the American Mosquito Control Association, v. 21, p. 328-330, 2005.

R DEVELOPMENT CORE TEAM. R: A language and environment for statistical computing. $R$ Foundation for Statistical Computing. 2017.

SEBASTIAN A, SEIN MM, THU MM, CORBET PS. Suppression of Aedes aegypti (Diptera, Culicidae) using augmentative release of dragonfly larvae (Odonata, Libellulidae) with community participation in Yangon, Myanmar. Bulletin of Entomological Research, v. 80, p. 223-232, 1990 .

SOARES CM, HAYASHI C, FARIA ACEA. Influência da disponibilidade de presas, do contraste visual e do tamanho das larvas de Pantala sp. (Odonata, Insecta) sobre a predação de Simocephalus serrulatus (Cladocera, Crustacea). Acta Scientiarum. Biological Sciences, v. 23, p. 357-362, 2008.

SUNAHARA T, ISHIZAKA K, MOGI M. Habitat size: a factor determining the oportunity for encounters between mosquito larvae and aquatic predators. Journal of Vector Ecology, v. 27, p. 8-20, 2002. 
TAUIL PL. Aspectos críticos do controle do dengue no Brasil. Cadernos de Saúde Pública, v. 18, p. 867-871, 2002.

WALKER ED, LAWSON DL, MERRITT RW, MORGAN WT, KLUG MJ. Nutrient dynamics, bacterial populations, and mosquito productivity in tree hole ecosystems and microcosms. Ecology, v. 72, p. 1529-1546, 1991.

WETERINGS R, UMPONSTIRA C, BUCKLEY H L. Predation rates of mixed instar Odonata naiads feeding on Aedes aegypti and Armigeres moultoni (Diptera: Culicidae) larvae. Journal of Asia-Pacific Entomology, v. 18, p. 1-8, 2015.

ZARET TM. Predation and freshwater communities. New Haven: Limnologia e Oceanografia, 1980. 393 p.

Recebido: $29 / 10 / 2019$

Aceito: 25/03/2020 